\title{
SAMSON: simulation supported traffic training environment
}

\section{Conference Paper}

Author(s):

Steinemann, Adrian; Kellenberger, Yves; Peikert, Pascal; Kunz, Andreas (D)

Publication date:

2011

Permanent link:

https://doi.org/10.3929/ethz-a-006397882

Rights / license:

In Copyright - Non-Commercial Use Permitted 


\title{
SAMSON: Simulation Supported Traffic Training Environment
}

\author{
Adrian Steinemann, Yves Kellenberger, Pascal Peikert, Andreas Kunz * \\ ICVR - Innovation Center Virtual Reality, Institute of Machine Tools and Manufacturing, ETH Zurich, Switzerland
}

\begin{abstract}
Since the publication of the first FTIR multi-touch interaction system [1], the public attention for the field of Single Display Groupware (SDG) has been rising constantly. Lately, former SDG systems with multi-interaction capabilities like DiamondTouch [2], reacTable [3], or Microsoft Surface [4] have been followed by promising systems, i.e. ThinSight [5] and MightyTrace [6]. The latter integrate their tracking technology into commercial liquid crystal displays (LCD), and thus drastically reduce the space requirements. Some recently published work [7] also conveys a trend to support industrial-oriented tasks as systems like BUILDIT [8] did some time ago.

We present a traffic training simulator concept based on discrete event simulation to ensure realistic traffic behavior, adequate visualization, and a user centered interaction concept on an SDG system to support training activities for policemen. Within this environment, policemen are able to train their behavior and the adequate choices under different traffic situations much like other professionals train their standard procedures, e.g. pilots in a flight simulator. Such a training environment will give the possibility to learn offline about important characteristics of intersections based on historical data like system stability, incident handling, or additional improvement potential.
\end{abstract}

KeYwords: Traffic Simulation, Discrete Event Simulation, Visualization, Human-Machine Interface.

INDEX Terms: H.1 [MODELS AND PRINCIPLES]: User/Machine Systems - Human factors; H.5 [INFORMATION INTERFACES AND PRESENTATION]: User Interfaces Training, help, and documentation, User-centered design, I.6 [SIMULATION AND MODELING]: Simulation Support Systems

\section{INTROduction AND USE CASE}

Today, big cities suffer from extensive traffic jams during the rush hours, when intersections get flooded with an immense quantity of traffic. These neuralgic points usually are handled with adaptive traffic lights, which react on the current situation supported by sensors and reaction algorithms. In the case of complex traffic flows based on a mix of public transportation, private transport, and extensive amounts of pedestrians, these static algorithms reach their limits. In this case, the traffic flow must be handled by policemen, who are able to react flexible and proactive to the occurring situation. The human being and his potential of comprehension and responsiveness in challenging situations excel the possibilities that can be supported with sophisticated traffic

\footnotetext{
* e-mail: \{steinemann, kunz\}@iwf.mavt.ethz.ch
} \{yvesk, peikertp\}@student.ethz.ch

IEEE International Symposium on Virtual Reality Innovation 2011 19-20 March, Singapore

978-1-4577-0037-8/11/\$26.00 @2011 IEEE light installations. However, the specialized policemen need the chance to get familiar with the circumstances of a particular intersection to ensure an optimal traffic flow.

The SAMSON traffic training environment envisions the possibility to train standard procedures within an offline and protected situation for traffic policemen. The focus is laid on an environment with the possibility to train the understanding of the overall system behavior from a comprehensive view without being restricted by the usual viewpoint during traffic handling. SAMSON therefore is based on a SDG system with an adequate interaction concept, a visualization of viewers' perspectives to compare the comprehensive view with restricted viewpoints on a vertical screen, and a discrete event simulation model running in the background to ensure realistic traffic behavior and its reactions to the user input (see Figure 1, left).
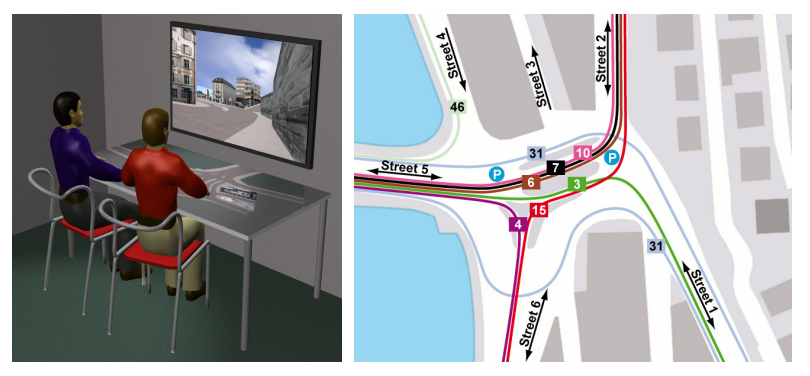

Figure 1. Left: SAMSON training environment, Right: Intersection with six streets, two policemen $(P)$, tram routes $(3,4,6,7,10$, $15)$, and bus lines $(31,46)$.

These components are interconnected and exchange data over the SAMSONintercom software, which is designed as central server and enables bidirectional communication between its clients: the discrete-event simulation suite (Plant Simulation [9]), the virtual environment (Blender Game Engine [10]), and the SDG system (MightyTrace [6]). The communication takes place as exchange of virtual objects, which are stored within a central repository, broadcasted to all connected clients, and stored within the clients' local repositories. Here, the updates are interpreted and lead to the needed reactions within the clients.

As a complex use case, we chose a plaza in Zurich, Switzerland. It is one of the main transportation hubs of the city and a neuralgic bottleneck. It involves public transportation, private transport, and lots of pedestrians. Six streets are connected through the plaza, here numbered from 1 to 6 (see Figure 1, right). The individual traffic is guided through the hub in a disrupted roundabout and must yield public transportation, pedestrians, and even traffic entering the roundabout at all times.

The plaza's situation gets problematic during the rush hours. As the traffic rises, the intersection needs to be controlled by two policemen on the main crossings. They decide which traffic gets the right of way and how many cars can cross the intersection before changing the precedence to the next axis. The policemen's goal is to ensure the cycle time and the correct sequence of the public transportation at all times. The second objective is to enable the maximum possible throughput of private traffic while keeping the waiting time for all participants as short as possible. 


\section{Simulation Model and Visualization}

The main focus of the simulation model was laid on the detailed reproduction of the traffic behavior. The model allows the policemen to make decisions which road gets the right of way and how many cars can pass. The public transportation on the other hand is modeled within an automatic reaction system with waiting queues. Each time the user changes the right of way, this reaction system is triggered and handles the control of waiting trams and busses with the possibility to cross the intersection based on the given right of way. After an extensive data acquisition, the model was verified within longtime simulations, and the basic behavior and distribution of traffic amounts were analyzed using the Sankey diagram function (see Figure 2, left).

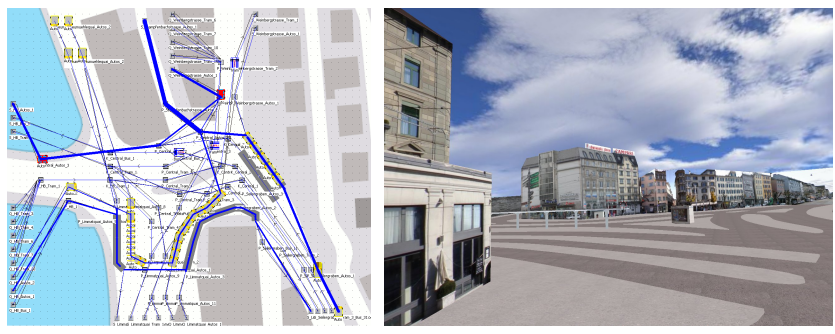

Figure 2. Left: Sankey diagram using Plant Simulation software [9] to verify the modeled behavior, Right: 3D model.

The subsequent validation started with an analysis of the system's settling time and a determination how many simulation runs are needed to reach an acceptable accuracy while keeping the computation time as low as possible. The results of the simulation were successfully compared to the acquired data of neuralgic points within the actual intersection. Besides this congruence, the determined throughput of cars and the preservation of the cycle time for the public transportation indicate that the model reproduces reality in an adequate manner and thus provides the basis for the intended traffic simulator.

With a network socket and location observers on all moving elements like cars, busses, and trams, the model is connected with SAMSONintercom and exchanges the object's position changes between the simulation model and the connected parts of the training environment like the 3D visualization or the SDG system.

The virtual representation of the actual intersection needs to be implemented as an interactive environment which shows all relevant occurrences and events of the intersection (e.g. location, public transportation, private transport, etc.). Using a game engine like the one of Blender [10], simulation tool events can be translated into movements within the virtual environment, and thus the simulation runs can be represented within a spatially correct representation (see Figure 2, right). Within SAMSON, this representation is used in a top view on the SDG system, and in a viewer's perspective on a vertical screen. This offers the possibility to perform virtual walkthroughs and learn about the visibility of intersection effects from different viewpoints.

\section{SDG SYSTEM AND INTERACTION CONCEPT}

As one of the keystones for SAMSON, we are now concentrating on an intuitive interaction and training concept based on the existing SDG environment. As the standard keyboard and mouse interaction within the SDG system is replaced with the usage of pens and Tangible User Interfaces (TUIs), this enables the user to concentrate on the tasks and objectives without being distracted by handling the system, also discussed by Stewart et al. [11]. Within SAMSON, the trainee will have to go through a three step training concept. The first step will be a 'see and understand' phase with TUIs for placing cameras to observe different restricted viewpoints and a time warping TUI to jump back and forth within the simulation to learn about the first signs of heavy traffic. The second step will be implemented as learning phase with the objective to train the actual intersection handling. This will be done with a traffic light-TUI and a right of way-TUI. With the traffic light-TUI, the trainee would be able to set parts of the intersection to an automated behavior. In the meantime, the trainee could take care of handling the traffic with the right of way TUI on other parts of the intersection. The trainee could improve step by step and work through different degrees of difficulty. Within the last phase of examination, the trainee will be put into stressful situations with extended traffic jams to check his abilities and to draw a comparison to other colleagues.

\section{Conclusion}

The presented simulation model is the centerpiece of the SAMSON traffic training environment. It will be connected over the existing intercommunication software with an SDG system, providing intuitive interaction concepts on a top view and an additional free camera view on a vertical screen. This environment targets the training of standard procedures for policemen handling the traffic on heavily loaded intersections. Next, we will concentrate on a three step training concept using tangible user interfaces to ease the use for the trainee and to offer the possibility to focus on the tasks and objectives of training.

\section{REFERENCES}

[1] Han, J. Y.: Low-Cost Multi-Touch Sensing through Frustrated Total Internal Reflection. In: Proc. of the 18th Annual ACM Symposium on User Interface Software and Technology, pp. 115 - 118. 2005.

[2] Dietz, P., Leigh, D.: DiamondTouch: A Multi-User Touch Technology. In: Proc. of the 14th Annual ACM Symposium on User Interface Software and Technology, pp. 219 - 226. 2001.

[3] Jordà, S., Geiger, G., Alonso, M., Kaltenbrunner, M.: The reacTable: Exploring the Synergy between Live Music Performance and Tabletop Tangible Interfaces. In: Proc. of the 1st Int. Conf. on Tangible and Embedded Interaction, pp. 139 - 146. 2007.

[4] Microsoft Corp. - Microsoft Surface, http://www.microsoft.com/surface/ (accessed December 2, 2010).

[5] Hodges, S., Izadi, S., Butler, A., Rrustemi, A., Buxton, B.: ThinSight: Versatile Multi-touch Sensing for Thin Form-factor Displays. In: Proc. of the 20th Annual ACM Symposium on User Interface Software and Technology, pp. 259 - 268. 2007.

[6] Hofer, R., Kaplan, P., Kunz, A.: MightyTrace: Multiuser Tracking Technology on LC-Displays. In: Proc. of the 26th Annual SIGCHI Conference on Human Factors in Computing Systems, pp. 215 218. 2008

[7] Zufferey, G., Jermann, P., Lucchi, A., Dillenbourg, P.: TinkerSheets: Using Paper Forms to Control and Visualize Tangible Simulations. In: Proc. of the 3rd Int. Conf. on Tangible and Embedded Interaction, pp. 377 - 384. 2009.

[8] Rauterberg, M., Fjeld, M., Krueger, H., Bichsel, M., Leonhardt, U., Meier, M.: BUILD-IT: A Planning Tool for Construction and Design. In: CHI 98 Conf. Summary on Human Factors in Computing Systems, pp. $177-178.1998$.

[9] Plant Simulation - Siemens PLM Software. http://www.plm.automation.siemens.com/en_us/products/tecnomatix /plant_design/plant_simulation.shtml (accessed December 1, 2010).

[10] Blender - Open Source 3D Content Creation Suite, http://www.blender.org/ (accessed December 2, 2010).

[11] Stewart, J., Raybourn, E. M., Bederson, B., and Druin, A.: When Two Hands Are Better Than One: Enhancing Collaboration Using Single Display Groupware. In: CHI 98 Conf. Summary on Human Factors in Computing Systems, pp. 287 - 288. 1998. 